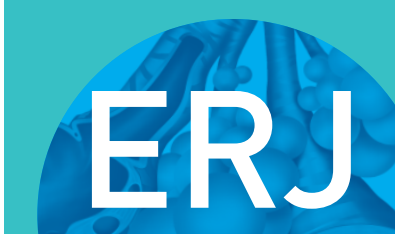

open research
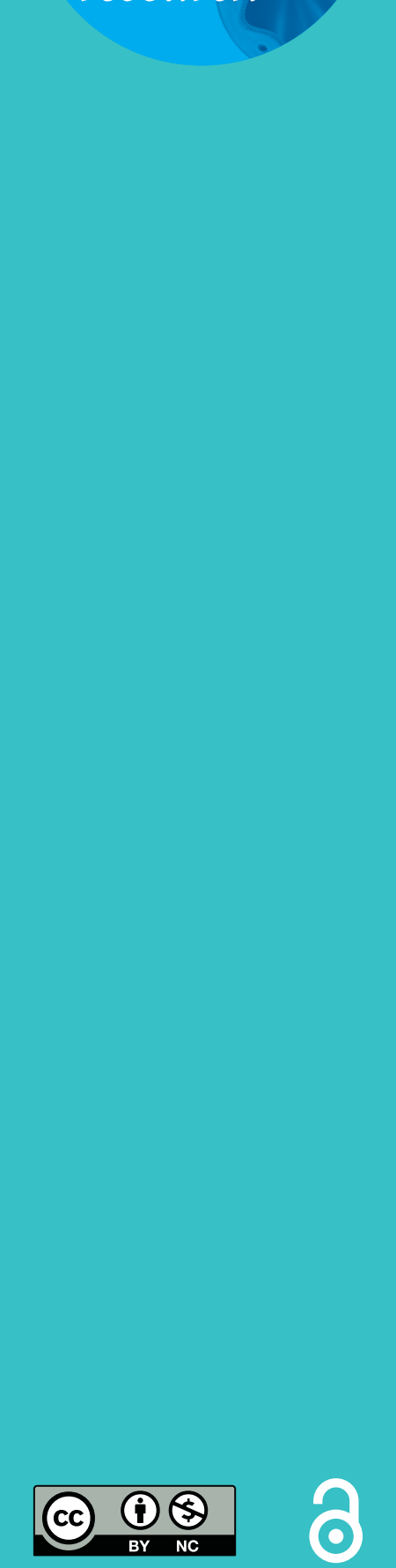

\section{Performance of transbronchial cryobiopsy in eccentrically and adjacently orientated radial endobronchial ultrasound lesions}

\author{
Sze Shyang Kho (D, Swee Kim Chan, Mei Ching Yong and Siew Teck Tie
}

Affiliation: Division of Respiratory Medicine, Dept of Medicine, Sarawak General Hospital, Ministry of Health, Kuching, Malaysia.

Correspondence: Sze Shyang Kho, Respiratory Medicine Unit (RCU), Sarawak General Hospital, Jalan Hospital 93586, Kuching, Sarawak, Malaysia. E-mail: bzk99ahotmail.com

\section{ABSTRACT}

Background: Radial endobronchial ultrasound (R-EBUS) is an effective technique in the diagnosis of peripheral pulmonary lesions (PPL). However, lesion orientation with regards to the radial probe remains an important factor for effective biopsy. "Within" orientation was associated with significantly higher diagnostic yield. Cryobiopsy is a novel technique in obtaining larger tissue samples with the frozen tip allowing biopsy in a $360^{\circ}$ direction, thus potentially achieving more effective biopsy in eccentrically and adjacently orientated lesions. We aimed to evaluate the performance and safety of transbronchial cryobiopsy versus forceps biopsy in eccentrically and adjacently orientated R-EBUS lesions.

Methods: Retrospective review of R-EBUS transbronchial biopsy for PPL over 17 months.

Results: 114 R-EBUS scans were included for analysis during the study period. Forceps biopsy was performed in $76(66.7 \%)$ cases and cryobiopsy in 38 (33.3\%) cases. Baseline demographics and lesion characteristics did not differ between the two groups. Median (interquartile range) lesion size was 3.48 $(2.63-4.51) \mathrm{cm}$. Overall, $41.2 \%$ of lesions were of eccentric orientation and $15.8 \%$ adjacent orientation; only $43 \%$ were concentric in orientation. Overall diagnostic yield was $67.5 \%$ (77 out of 114). Orientation remained an important factor affecting diagnostic yield. Transbronchial cryobiopsy significantly increased the diagnostic yield in eccentrically and adjacently orientated lesions to $75.0 \%$ (18 out of 24), compared to $48.8 \%$ (20 out of 41 ) obtained via forceps biopsy ( $\mathrm{p}<0.05$ ); but not in concentric lesions. Cryobiopsy was associated with more mild and moderate bleeding complications compared to the forceps biopsy group.

Conclusions: Transbronchial cryobiopsy under R-EBUS guidance is a safe procedure which potentially increases diagnostic yield in eccentrically and adjacently orientated PPLs.

@ERSpublications

Transbronchial cryobiopsy under R-EBUS guidance potentially increases diagnostic yield in eccentric and adjacently orientated peripheral pulmonary lesions. http://bit.ly/2KWtkGq

Cite this article as: Kho SS, Chan SK, Yong MC, et al. Performance of transbronchial cryobiopsy in eccentrically and adjacently orientated radial endobronchial ultrasound lesions. ERJ Open Res 2019; 5: 00135-2019 [https://doi.org/10.1183/23120541.00135-2019].

Received: 31 May 2019 | Accepted after revision: 13 Aug 2019

Copyright $\odot E R S$ 2019. This article is open access and distributed under the terms of the Creative Commons Attribution Non-Commercial Licence 4.0. 


\section{Introduction}

Pulmonary nodules can be detected in $20-50 \%$ of individuals in regions that have established low-dose computed tomography (CT) screening programmes for populations at high risk of lung cancer [1]. Although the majority of these nodules are benign, a significant number are malignant; diagnosis and effective treatment led to a $20 \%$ reduction in mortality [2]. Moreover, in regions with high tuberculosis incidence, a significant number of the benign nodules would be proven to be tuberculosis, a public health and infection control concern, which also require early diagnosis and treatment [3]. However, despite the high demand for histological diagnosis, sampling of peripheral pulmonary lesions (PPL) remains a challenge. Guided bronchoscopy has been gaining popularity among pulmonologists due to its lower risk of pneumothorax compared to CT-guided transthoracic needle aspiration.

Radial endobronchial ultrasound (R-EBUS) with guide sheath provides a reasonable diagnostic yield for PPL [4]. However, the diagnostic yield still very much depends on the orientation of PPL to the radial probe [5]. Conventional biopsy forceps allow only forward advancement, posing a difficulty in adjacently orientated PPL, even when the lesion can be identified by R-EBUS, as lateral biopsy is not possible. For eccentric lesions, when the placement of probe is biased to the lesion edge only, inadequate depth of the forceps biopsy may also lead to inconclusive results. Cryobiopsy is a novel biopsy tool which is capable of lateral biopsy [6]. When the cryoprobe is activated, it allows tissue to be obtained in a $360^{\circ}$ manner laterally from the tip of the probe. This advantage of lateral biopsy may increase the diagnostic yield of eccentrically and adjacently orientated R-EBUS lesions compared to conventional forceps biopsy. Transbronchial cryobiopsy has been employed in the diagnosis of diffuse parenchymal lung disease (DPLD); however, its use in the biopsy of PPL has been scarcely reported in literature.

Our study aimed to evaluate the performance of transbronchial cryobiopsy in eccentrically and adjacently orientated R-EBUS lesions in comparison to forceps biopsy. In addition, we aimed to explore the overall diagnostic yield of R-EBUS guided transbronchial lung biopsy for PPL and evaluate the overall baseline demographic, target lesion, procedure characteristic and complication rate.

\section{Materials and methods}

\section{Setting}

This study was conducted in the respiratory care unit, Sarawak General Hospital, Malaysia. Sarawak General Hospital is a 900-bed hospital which is the only tertiary referral centre for respiratory diseases in the state of Sarawak. The respiratory medicine unit performs $\sim 600$ respiratory endoscopies per year, including advanced diagnostic and therapeutic bronchoscopies.

\section{Study design}

This study retrospectively reviewed all adult patients undergoing R-EBUS-guided transbronchial biopsy for PPL in the respiratory care unit in Sarawak General Hospital over 17 months (December 1, 2016 to April 30, 2018). Patients with endobronchial lesions biopsied during initial airway examination, as well as patients with incomplete clinical data or information were excluded. Patients with PPL not visualised by R-EBUS were also excluded. The study protocol was approved by the medical research and ethics committee, Ministry of Health Malaysia (NMRR-17-2834-39164 IIR).

\section{Pre-procedural planning}

For pre-procedural planning, all patients from our centre underwent a thin-slice ( $1 \mathrm{~mm}$ slice thickness) CT scan. However, scans were not repeated in patients referred from other centres with a CT scan, regardless of the slice thickness or reconstruction protocol, due to resource constraints. Pre-procedure airway mapping was performed using the free open-source DICOM software (RadiAnt DICOM viewer version 5.0.0; Medixant, Poznań, Poland) by analysing subsegmental airway anatomy in multiplanar reconstruction from the CT scan in axial, coronal and sagittal planes. Bronchial branch reading was then performed by meticulously analysing the bronchus sign and bronchial bifurcations that lead into the target lesion. The planned road map representing actual bronchoscopic vision of the segmental airway leading into the target lesion was then drawn by hand in a schematic diagram. No additional navigational software was used in this study to assist with procedural planning. This bronchial branch reading technique has been well described in literature $[7,8]$.

\section{Bronchoscopy and R-EBUS procedure}

The procedures were performed either by one consultant pulmonologist (S.T. Tie) with 10 years' experience of flexible and rigid bronchoscopy, or by pulmonology fellows under direct supervision of the consultant pulmonologist. Flexible bronchoscopies were performed under conscious sedation or total intravenous anaesthesia as per our unit protocol. Decision for advanced airways (i.e. rigid bronchoscope or 
endotracheal intubation) was at the discretion of the endoscopist after considering the risk and benefit of the procedure to the patient. All patients had signed and dated informed consent prior to procedure.

Various flexible diagnostic and therapeutic bronchoscopes (BF-1TH190, BF-Q180, BF-P190; Olympus Medical, Tokyo, Japan; EB-530XT Fujinon, Tokyo, Japan) were used for the procedures. Olympus R-EBUS system (Olympus MAJ-1720 and EU-ME2 Premier Plus) were used in all cases of R-EBUS procedure. After ruling out endobronchial lesions, the $20 \mathrm{~Hz} 2.0 \mathrm{~mm}$ or $1.7 \mathrm{~mm}$ R-EBUS probe (Olympus Medical UM-S20-20R, UM-S20-17S) within a guide sheath (Olympus Medical SG-201C, SG-200c) was inserted into the pre-planned target segment via the bronchoscope working channel. Upon identification of the lesion via R-EBUS, the guide sheath was locked in place and R-EBUS probe removed. In selected cases, guide sheath was not used and subsequent placement of biopsy tools was guided by single planar fluoroscopy.

For transbronchial forceps biopsy, a standard fenestrated cup flexible forceps (FB231-D, FB233-D, Olympus Medical) was introduced into the guide sheath, and five biopsies were taken during each round of the procedure; the lesion location was reaffirmed with R-EBUS via guide sheath if further rounds of biopsy were required. For cryobiopsy, a $1050 \mathrm{~mm} 1.9 \mathrm{~mm}$ cryoprobe was used in conjunction with the ERBECRYO ${ }^{\circ} 2$ system (ERBE, Medizintechnik, Tübingen, Germany). An endobronchial balloon (B5-2C; Olympus Medical) was place at the target segmental bronchi after lesion localisation by R-EBUS if the advanced airways were used during the procedure. The cryoprobe was subsequently inserted into the guide sheath, activated for 3-4 s and removed en bloc with the bronchoscope from the patient. This retrieval process was done rapidly while maintaining cryoprobe activation until the bronchoscope and cryoprobe were completely out from the patient, either from an artificial or natural airway orifice. The pre-placed balloon was inflated immediately upon removal of bronchoscope. Tissue from cryobiopsy was thawed and retrieved in normal saline from the cryoprobe.

Biopsied specimens were fixed in formalin solution and sent to our pathology lab immediately for processing and analysis. Bronchoscopy was performed again to look for evidence of airway bleeding and other complications after each round of biopsy. Barring any complication, the procedure was repeated again until adequate tissue was obtained. After biopsy, $50 \mathrm{~cm}^{3}$ of normal saline was instilled into the target segment via guide sheath and aspirated for microbiological or cytological examination in cases where infective causes were suspected. Post-procedure, a chest radiograph was performed to look for pneumothorax.

The target lesion was classified as "concentric" when the probe was within and completely surrounded by the lesion, and "eccentric" when the probe was within but largely biased toward one side at the edge of the lesion [9]. The target lesion was classified as "adjacent" when the probe was not within the lesion and only placed next to the lesion (figure 1).

Bleeding was categorised into mild, moderate and severe. Mild bleeding required only suction to clear, with or without local instillation of adrenaline; moderate bleeding involved prolonged balloon blockade; severe bleeding events required additional intervention, such as bronchial artery embolisation, transfusion, intubation or emergency surgical treatment [10].

The procedure was considered to be conclusive if histological, microbiological or cytological studies provided definitive results, allowing specific treatment to be implemented. Procedures were considered to be inconclusive if results failed to provide a definitive answer to the presenting clinical problem, requiring further intervention or biopsy.
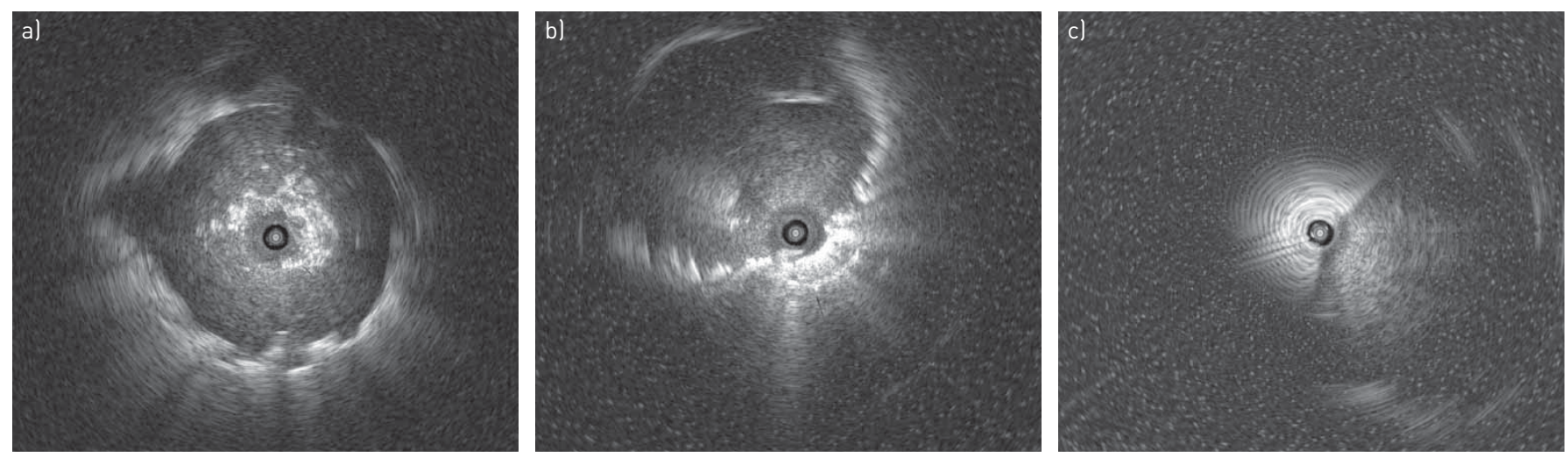

FIGURE 1 Radial endobronchial ultrasound orientation. a) Concentric orientation; b) eccentric orientation; c) adjacent orientation. 


\section{Statistical analysis}

Data analysis was performed using SPSS (version 20; Chicago, IL, USA). For variables with assumed normal distribution, data were presented as mean $\pm \mathrm{SD}$, whereas for variables with non-normal distribution, data was presented as median (interquartile range (IQR)). Categorical data were expressed as absolute numbers and percentages. Between the two groups (cryobiopsy and forceps biopsy), comparisons of baseline data were performed using the independent sample t-test for variables with assumed normal distribution; and with Mann-Whitney test for non-normal distribution variables. All categorical variables were compared between the two groups using the Pearson's Chi-squared test or Fisher's exact test. A value of $\mathrm{p}<0.05$ was considered statistically significant.

\section{Results}

During the study period, a total of 127 R-EBUS procedures were performed. 13 cases were excluded: three cases due to presence of endobronchial lesion, six cases due to failure of localisation via R-EBUS and four R-EBUS-guided biopsy cases performed for DPLD rather than PPL. 114 cases were included for analysis. Sample cases are presented in figures 2 and 3.

\section{Baseline clinical and target lesion characteristics}

Forceps biopsy was performed in $76(66.7 \%)$ cases and cryobiopsy in 38 (33.3\%) cases. Our cohort comprised of mainly male patients with overall median (IQR) age of 58.5 (49.8-68.3) years. Overall lesion size was 3.48
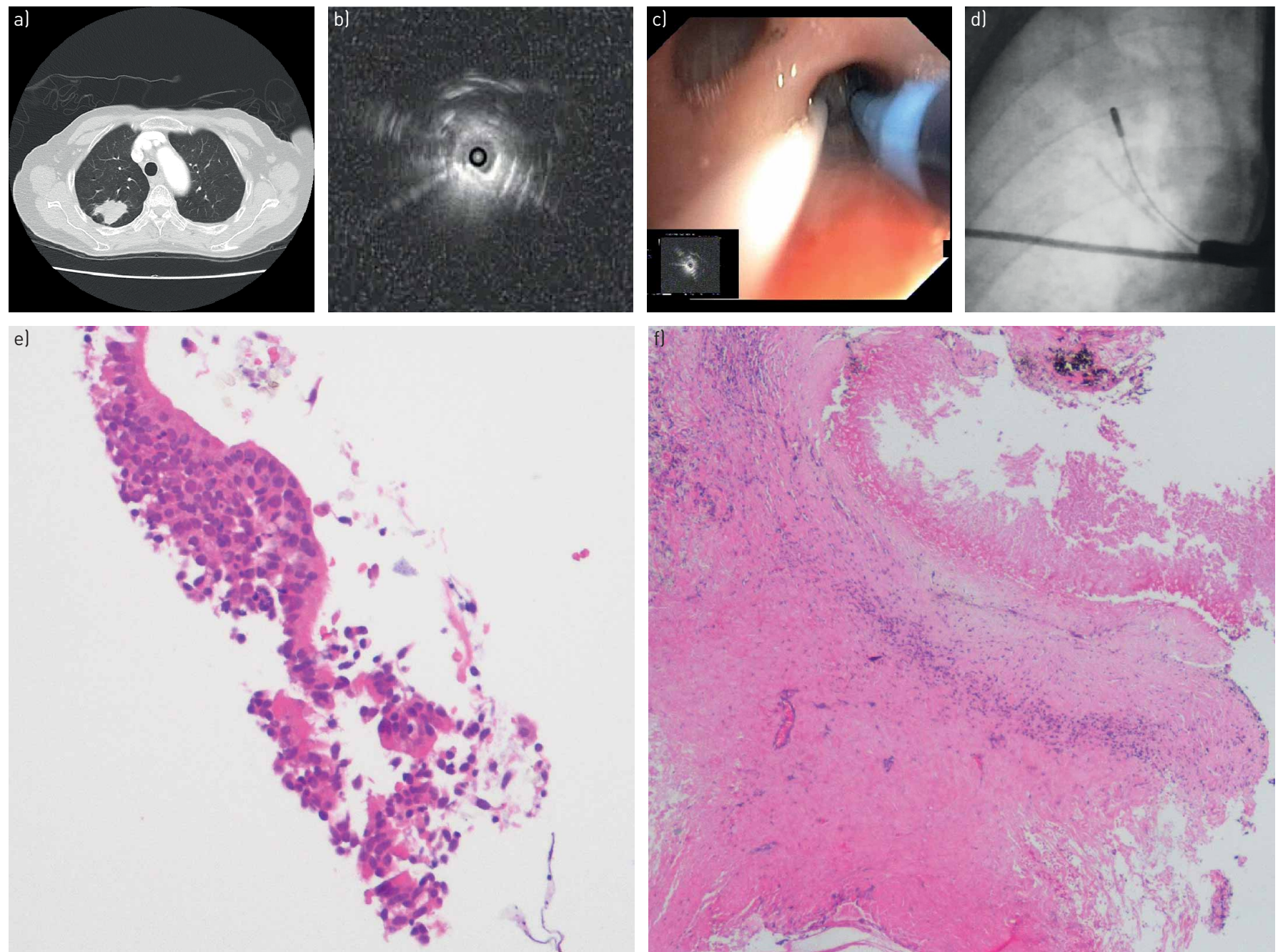

FIGURE 2 Representative case 1. a) Axial computed tomography thorax demonstrates a $2.2 \mathrm{~cm}$ peripheral pulmonary lesion over right upper lobe posterior segment; b) radial endobronchial ultrasound revealed an eccentrically orientated lesion; c) placement of $1.9 \mathrm{~mm}$ cryoprobe with prophylactic blocking balloon at the ostium of posterior segment of right upper lobe; d) single planar fluoroscopy demonstrating placement of cryoprobe (without guide sheath) and blocking balloon; e) forceps biopsy revealing only bronchial epithelium (haematoxylin and eosin stain (HE), 100 -fold magnification); f) cryobiopsy revealing chronic granulomatous inflammation suggestive of tuberculosis (HE, 400-fold magnification). 

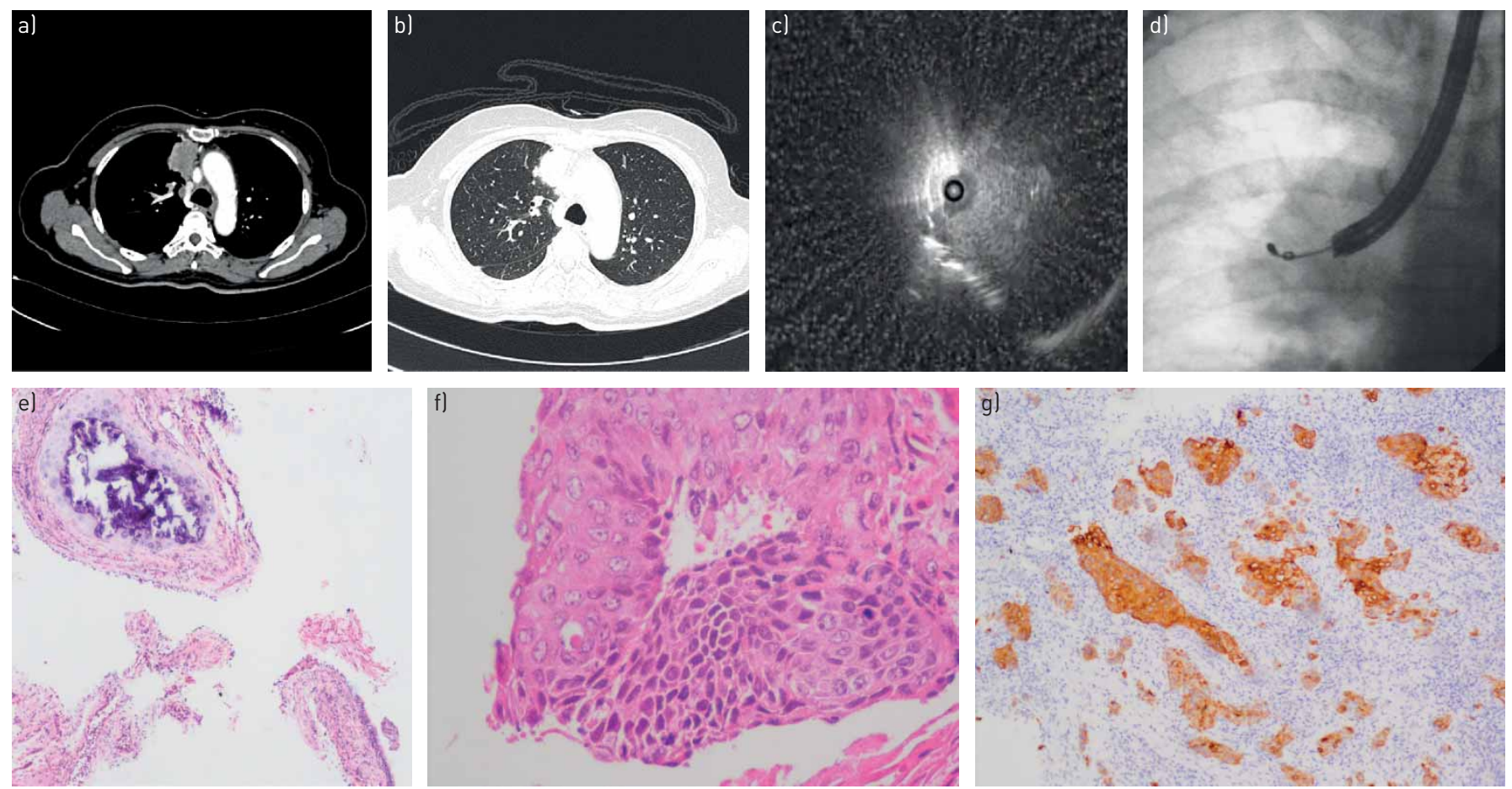

FIGURE 3 Representative case 2. a,b) Axial computed tomography thorax demonstrates a solitary pulmonary nodule at anterior segment of right upper lobe in lung and mediastinal window; c) adjacently orientated radial endobronchial ultrasound lesion at target segment; d) placement of cryoprobe via guide sheath into the lesion via fluoroscopy guidance; e) forceps biopsy revealing only superficial peribronchial tissue with cartilage (haematoxylin and eosin stain (HE), $\times 40$-fold magnification); f,g) cryobiopsy confirmed squamous cell carcinoma with positive cytokeratin (CK) $5 / 6$ staining (f, HE, 400-fold magnification; g, CK 5/6 stain, 100-fold magnification).

$(2.63-4.51) \mathrm{cm}$ with the majority of the lesions located in the outer third of the hemithorax, and more commonly in the upper lobe. 57\% (65 out of 114) of the lesions demonstrated eccentric and adjacent orientations to the R-EBUS probe, with only $43 \%$ (49 out of 114) in concentric orientations. The baseline clinical and target lesion characteristics did not differ between the forceps and cryobiopsy groups (table 1).

\section{Procedure characteristics}

Overall median (IQR) procedure time was $45(35.8-60.0)$ min, with cryobiopsy recording a significantly longer duration at $50 \mathrm{~min}$ compared to $40.5 \mathrm{~min}$ in forceps biopsy. The majority of procedures (79.8\%) were performed without advanced airway. However, advanced airway was used more in the cryobiopsy group, to allow placement of prophylactic balloon in high-risk cases. Moreover, use of fluoroscopy was more common in the cryobiopsy group. However, guide sheath was used significantly less in the cryobiopsy group at only $57.9 \%$ versus $94.7 \%$ in the forceps group. Details of procedures are listed in table 1 .

\section{Overall diagnostic yield}

Overall diagnostic yield of our cohort was $67.5 \%$ (77 out of 114). Diagnostic yields for concentrically $(n=49)$, eccentrically $(n=47)$ and adjacently $(n=18)$ orientated lesions were $79.6 \%, 63.8 \%$ and $44.4 \%$, respectively. Concentric lesions recorded a higher diagnostic yield compared to eccentrically and adjacently orientated lesions (79.6\% (39 out of 49 ) versus $58.5 \%$ (38 out of 65 ); $\mathrm{p}<0.05$ ).

\section{Performance of transbronchial cryobiopsy in eccentrically and adjacently orientated R-EBUS lesions $(n=65)$}

For concentric lesions $(\mathrm{n}=49)$, cryobiopsy did not increase the diagnostic yield significantly compared to the forceps biopsy group (85.7\% (12 out of 14 ) versus $77.1 \%$ ( 27 out of 35 ); $\mathrm{p}=0.501$ ). For eccentric lesions $(\mathrm{n}=47)$, the diagnostic yield was 56.3\% (18 out of 32$)$ in forceps biopsy and $80 \%$ (12 out of 15$)$ in cryobiopsy ( $\mathrm{p}=0.114)$. For adjacently orientated lesions $(\mathrm{n}=18)$, cryobiopsy increased the diagnostic yield to $66.7 \%$ (six out of nine) from $22.2 \%$ (two out of nine) in forceps biopsy ( $\mathrm{p}=0.058$ ).

Overall, for eccentrically and adjacently orientated lesions, cryobiopsy significantly increased the diagnostic yield from $48.8 \%$ (20 out of 41 ) to $75.0 \%$ (18 out of 24$)$ ( $p<0.05$ ) (figure 4 ). 


\begin{tabular}{|c|c|c|c|c|}
\hline & Overall & Forceps biopsy & Cryobiopsy & p-value \\
\hline Subjects & 114 & 76 & 38 & \\
\hline \multicolumn{5}{|l|}{ Patient characteristics } \\
\hline Age years & $58.5(49.8-68.3)$ & $60(50-70)$ & $56(47.8-64.5)$ & 0.210 \\
\hline \multicolumn{5}{|l|}{ Sex } \\
\hline Male & $78(68.4)$ & $53(69.7)$ & $25(65.8)$ & \multirow[t]{2}{*}{0.669} \\
\hline Female & $36(31.6)$ & 23 (30.3) & 13 (34.2) & \\
\hline \multicolumn{5}{|c|}{ Target lesion characteristics } \\
\hline Size $\mathrm{cm}$ & $3.48(2.63-4.51)$ & $3.50(2.65-4.81)$ & $3.39(2.59-4.19)$ & 0.637 \\
\hline \multicolumn{5}{|l|}{ Lobe } \\
\hline Upper lobe & $69(60.5)$ & $46(60.5)$ & $23(60.5)$ & \multirow[t]{3}{*}{0.976} \\
\hline Middle lobe/lingular & $16(14.1)$ & $11(14.5)$ & $5(13.2)$ & \\
\hline Lower lobe & $29(25.4)$ & $19(25.0)$ & $10(26.3)$ & \\
\hline \multicolumn{5}{|l|}{ Location } \\
\hline Inner third & 20 (17.5) & 15 (19.7) & $5(13.2)$ & \multirow[t]{3}{*}{0.172} \\
\hline Middle third & 45 (39.5) & $33(43.4)$ & $12(31.6)$ & \\
\hline Outer third & $49(43.0)$ & $28(36.8)$ & $21(55.3)$ & \\
\hline \multicolumn{5}{|l|}{ Orientation } \\
\hline Concentric & $49(43.0)$ & $35(46.1)$ & $14(36.8)$ & \multirow[t]{3}{*}{0.248} \\
\hline Eccentric & $47(41.2)$ & $32(42.1)$ & 15 (39.5) & \\
\hline Adjacent & $18(15.8)$ & 9 (11.8) & $9(23.7)$ & \\
\hline \multicolumn{5}{|l|}{ Procedure characteristics } \\
\hline Time min & $45(35.8-60)$ & $40.5(35-51.5)$ & $50.0(45-60)$ & $<0.01$ \\
\hline \multicolumn{5}{|l|}{ Airway } \\
\hline Transnasal & 91 (79.8) & 69 (90.8) & 22 (57.9) & \multirow[t]{2}{*}{$<0.001$} \\
\hline Advanced airway & $23(20.2)$ & 7 (9.2) & $16(42.1)$ & \\
\hline Fluoroscopy & $91(79.8)$ & 56 (73.7) & $35(92.1)$ & $<0.05$ \\
\hline Guide sheath & 94 (82.5) & 72 (94.7) & 22 (57.9) & $<0.001$ \\
\hline
\end{tabular}

Data are presented as $n$, median (interquartile range) or $n(\%)$, unless otherwise stated. Bold type represents statistical significance.

63.2\% (24 out of 38 ) of our cryobiopies were performed in eccentrically and adjacently orientated lesions. Of these 24 cases, forceps biopsy was performed in addition to cryobiopsy in $66.7 \%$ (16 out of 24). Of these 16 cases, only cryobiopsy yielded a conclusive diagnosis in $43.8 \%$ (seven out of 16 ). In $31.2 \%$ (five out of 16), both forceps and cryobiopsy yielded a conclusive diagnosis, and in $25.0 \%$ (four out of 16), both groups failed to achieve a diagnostic yield. Although usage of advanced airway, fluoroscopy and guide sheath differ between these two groups, analysis showed that these factors did not affect the diagnostic yield in the eccentrically and adjacently orientated groups (table 2).

The majority of conclusive histologies in the eccentrically and adjacently orientated groups were malignant disease at $57.9 \%$, while infective diseases made up 39.5\%. Interestingly, 57.2\% of the nondiagnostic forceps biopsies in this group yielded only bronchial epithelium, probably due to inadequate depth of biopsy. The details of the histological findings are displayed in table 3.

\section{Complications}

Only one pneumothorax occurred in the forceps biopsy group with no pneumothorax in the cryobiopsy group; hence, the overall pneumothorax rate was $0.8 \%$ in our current cohort. However, mild and moderate bleeding complications were more common in the cryobiopsy group compared to forceps biopsy group ( $47.4 \%$ versus $7.9 \%, \mathrm{p}<0.001)$. However, most bleeding episodes in cryobiopsy were mild $(39.5 \%)$, requiring only suction with local adrenaline instillation; while moderate bleeding occurred in three (7.9\%) patients. There was no occurrence of severe life-threatening bleeding event.

\section{Discussion}

Various guided modalities are available to improve bronchoscopic access to PPL, as diagnostic yield would otherwise be $<20 \%$ in routine unguided bronchoscopies for PPL [4]. R-EBUS-guided transbronchial lung biopsy of PPL is a novel technique with meta-analysis revealing overall diagnostic yield of $70.6 \%$ with a low complication rate of $2.8 \%$ [5]. Our cohort reported an overall slightly lower diagnostic yield of $67.5 \%$, which is probably due to the significant proportion (57\%) of eccentrically and adjacently orientated lesions in our cohort. 


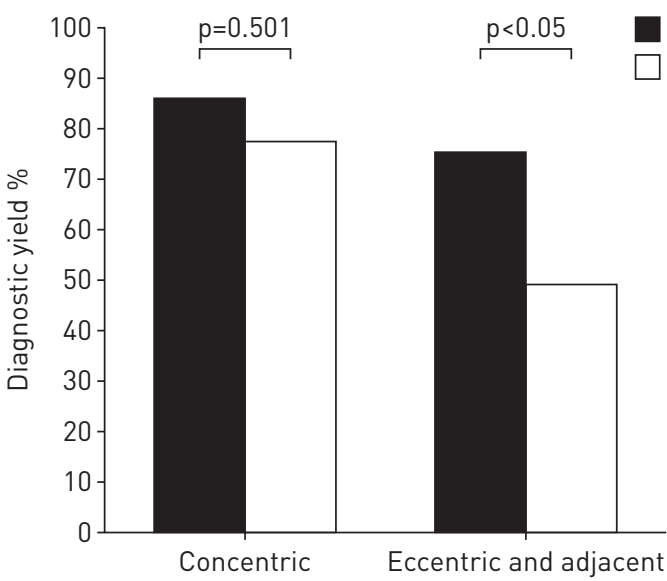

FIGURE 4 Performance of transbronchial cryobiopsy versus forceps biopsy in concentrically orientated versus eccentrically and adjacently oriented lesions.

Despite the usage of various guidance techniques, diagnostic yield of PPL is still potentially dependent on biopsy methods [6]. Conventional forceps biopsy frequently results in superficial, small and crushed specimens, which may not be suitable or adequate for immunohistochemical or molecular studies. Cryobiopsy is a novel technique available for bronchoscopic biopsy. Its use in endobronchial biopsy has been proven to be safe and provides superior diagnostic yield compared to forceps biopsy [11]. Furthermore, transbronchial cryobiopsy in DPLD has been established in terms of safety, with lower complication rates compared to surgical lung biopsy [12]. However, literature on transbronchial lung cryobiopsy for the diagnosis of PPL is scarce and limited [13]. Schunmann et al. [6] had demonstrated in the largest series to date that transbronchial cryobiopsy of PPL with R-EBUS guidance is safe and feasible in 31 patients. Hibare et al. [14] also performed transbronchial cryobiopsy in 28 patients with a diagnostic yield of $67.9 \%$. Since then, other smaller case series have demonstrated encouraging diagnostic yield and safety profiles $[15,16]$.

A proposed advantage of cryobiopsy is the ability of the cryoprobe to obtain a spherical core of tissue surrounding the tip when it is frozen, thus allowing lateral biopsy, which increases the chance of successful biopsy in lesions in adjacent orientation to the probe; as compared to conventional forceps biopsy, which only allows biopsy in a forward direction $[6,16]$. It is recognised that in lesions adjacent to the airway, mucosal invasion frequently does not occur, resulting in low diagnostic yield of bronchoscopic forceps biopsy $[17,18]$. Our study explored this proposed advantage and demonstrated that cryobiopsy indeed significantly increased the diagnostic yield of eccentrically and adjacently orientated lesions. The lateral

\section{TABLE 2 Factors influencing diagnostic yield in eccentric and adjacent lesions}

\begin{tabular}{lcc} 
& Diagnostic yield & p-value \\
\hline Biopsy method & & \\
$\quad$ Forceps biopsy & $48.8(20 / 41)$ & $\mathbf{0 . 0 5}$ \\
$\quad$ Cryobiopsy & $75.0(18 / 24)$ & \\
Lesion size & $56.7(17 / 30)$ & 0.786 \\
$<3 \mathrm{~cm}$ & $60.0(21 / 35)$ & \\
$\geqslant 3 \mathrm{~cm}$ & $54.9(28 / 51)$ & 0.266 \\
Airway & $71.4(10 / 14)$ & \\
$\quad$ Transnasal & $59.6(31 / 52)$ & 0.706 \\
$\quad$ Advanced airway & $53.8(7 / 13)$ & \\
Fluoroscopy & $54.7(29 / 53)$ & 0.198 \\
$\quad$ With & $75.0(9 / 12)$ & \\
$\quad$ Without & \\
Guide sheath & \\
$\quad$ With & & \\
Without & & \\
\hline Data are presented as $\%$ (n/N), unless otherwise stated. $\mathrm{n}=65$. Bold type represents statistical significance.
\end{tabular}




\section{TABLE 3 Histologies for all conclusive lesions in the eccentric and adjacent groups}

Overall Forceps biopsy Cryobiopsy

\begin{tabular}{|c|c|c|c|}
\hline Conclusive histology & $38(58.5)$ & $20(48.8)$ & $18(75.0)$ \\
\hline \multicolumn{4}{|l|}{ Malignant } \\
\hline Adenocarcinoma lung & $17(44.8)$ & $9(45.0)$ & $8(44.4)$ \\
\hline Squamous cell carcinoma & $4(10.5)$ & $3(15.0)$ & $1(5.6)$ \\
\hline Metastatic sarcoma & $1(2.6)$ & $1(5.0)$ & $0(0.0)$ \\
\hline \multicolumn{4}{|l|}{ Benign } \\
\hline Nodular lymphoid hyperplasia & $1(2.6)$ & $0(0.0)$ & $1(5.6)$ \\
\hline \multicolumn{4}{|l|}{ Infection } \\
\hline \multicolumn{4}{|l|}{ Tuberculosis } \\
\hline Granulomatous inflammation & 9 (23.8) & $3(15.0)$ & 6 (33.3) \\
\hline Necrotic tissue with guide sheath positive for Xpert MTB/RIF & $1(2.6)$ & $1(5.0)$ & $0(0.0)$ \\
\hline $\begin{array}{l}\text { Chronic inflammation with therapeutic response to } \\
\text { antituberculous drug }\end{array}$ & $4(10.5)$ & $2(10.0)$ & $2(11.1)$ \\
\hline $\begin{array}{l}\text { Chronic inflammation with guide sheath culture positive for } \\
\text { Penicillium }\end{array}$ & $1(2.6)$ & $1(5.0)$ & $0(0.0)$ \\
\hline Inconclusive histology & $27(41.5)$ & $21(51.2)$ & $6(25.0)$ \\
\hline \multicolumn{4}{|l|}{ Nondiagnostic } \\
\hline Bronchial epithelium & $13(48.2)$ & 12 (57.2) & $1(16.7)$ \\
\hline Chronic inflammation & 5 (18.5) & $3(14.2)$ & 2 (33.2) \\
\hline Fibrotic alveolar septa & $2(7.4)$ & $1(4.8)$ & $1(16.7)$ \\
\hline Necrotic tissue & $2(7.4)$ & $1(4.8)$ & $1(16.7)$ \\
\hline Fibrocollagenous tissue & $1(3.7)$ & $1(4.8)$ & $0(0.0)$ \\
\hline Haemorrhagic lung tissue & $3(11.1)$ & 3 (14.2) & $0(0.0)$ \\
\hline Entrapped gland & $1(3.7)$ & $0(0.0)$ & $1(16.7)$ \\
\hline
\end{tabular}

advantage of cryobiopsy is further driven home, when we find that $57.2 \%$ of inconclusive forceps biopsies in eccentrically and adjacently orientated lesions were actually bronchial epithelium, probably due to superficial inadequate depth of lateral biopsy. Although Schunmann et al. [6] had reported that cryobiopsy did not increase their diagnostic yield significantly, the majority (64.5\%) of the lesions in their cohort had "within" orientation and only $35.5 \%$ were adjacently orientated, which may explain the insignificant increment of diagnostic yield by cryobiopsy. Similarly, in our cohort, the use of cryobiopsy in concentric lesions did not increase the diagnostic yield significantly. Despite reports that usage of peripheral transbronchial needle aspiration of PPL is feasible in adjacently orientated lesions, this might be technically challenging as well as limited by small specimen size [19].

Cryobiopsy is not without challenges. Firstly, cryoprobe size and stiffness hinder the usage of guide sheath in the cryobiopsy group, with only $57.9 \%$ allowing guide sheath usage in our cohort. Usage of guide sheath in PPL biopsy is essential, as meta-analysis has proven guide sheath to be an important factor in ensuring accurate, consistent biopsy after successful R-EBUS navigation [4]. HERATH and YAP [15] demonstrated the importance of guide sheath in four out of six patients who underwent cryobiopsy of PPL via guide sheath after localisation by R-EBUS. Secondly, the need for renavigation after each round of cryobiopsy poses a challenge to the overall procedure, as the cryoprobe and bronchoscope need to be removed en bloc after each round of cryobiopsy. Our cohort revealed a longer procedural time for cryobiopsy in comparison to forceps biopsy. A smaller calibre flexible cryoprobe within a sheath, which allows specimen retrieval through the working channel of the bronchoscope, which has recently shown good results in animal studies, may address this challenge in the future [20]. Thirdly, bleeding is common and always a concern in cryobiopsy [11]. Expert groups have recommended that cryobiopsy in DPLD not be performed too proximally in the middle third of the lung due to the presence of medium-sized vessels, which are not completely protected by cartilage plate [10]. However, PPLs are not confined to these zones as compared to DPLD and the majority of lung adenocarcinoma are predominantly peripheral in origin [17]. Encouragingly, BERIM et al. [21] have demonstrated that the simultaneous usage of R-EBUS and fluoroscopy could potentially decrease bleeding complications associated with cryobiopsy in DPLD patients by identifying vessels at the site of cryobiopsy. In their cohort, one in four patients suffered a massive haemorrhage without R-EBUS guidance, while no massive bleeding was observed in all six patients in whom cryobiopsy was performed with R-EBUS guidance. In addition, SchuHmann et al. [6] reported a low bleeding rate without prophylactic balloon placement in their PPL cryobiopsy cohort, 
which may be due to the combined use of R-EBUS and guide sheath in detection of the target lesion prior to sampling. Although bleeding complication was more common in our cryobiopsy group, all the episodes were mild, and through the use of R-EBUS prior to biopsy, no massive bleeding episode was encountered. Our finding was in agreement with these reports that bleeding risk may not be excessive in cryobiopsy of PPL with R-EBUS guidance, and extrapolation of DPLD cryobiopsy protocol into PPL sampling with R-EBUS guidance may not be appropriate. However, further prospective studies are required to evaluate the actual bleeding risk of PPL cryobiopsy under R-EBUS guidance.

There are several limitations to our study. Firstly, it is a retrospective, single-institution experience. Hence, our results may not be generalisable to other populations. Furthermore, our study design is compounded by selection bias, as there was no randomisation of procedures between the two groups. Hence, the association of better diagnostic yields from transbronchial cryobiopsy in eccentrically and adjacently orientated R-EBUS lesion cannot be fully determined. However, this retrospective observational study provides a new insight to the current dilemma in bronchoscopic sampling of eccentrically and adjacently orientated R-EBUS lesions. Further prospective randomised studies are needed. Secondly, our procedures were heterogeneous as there was no standardised procedural protocol. One factor includes the various types of bronchoscopes (different calibre) used in our study. Conversely, as only those lesions identifiable by R-EBUS were included for analysis, the bronchoscope with or without guide sheath would have acted only as a conduit for biopsy, whereas our biopsy methods after localisation (either forceps or cryobiopsy) were consistent throughout; thus we believe that the study result remains representative. Furthermore, although both groups differed in usage of fluoroscopy, guide sheath and advanced airways, our analysis shows that these factors did not affect the diagnostic yield significantly in eccentrically and adjacently orientated lesions (table 2). Thirdly, we did not report the cumulative diagnostic yield of forceps biopsy and cryobiopsy for every single lesion, and the sequence of biopsy method for each lesion was not taken into consideration. However, in a subgroup analysis of 24 cases where both forceps biopsy and cryobiopsy were performed, only cryobiopsy was able to establish a diagnosis in $43.8 \%$ of cases.

In conclusion, this study demonstrated that transbronchial cryobiopsy for PPL under R-EBUS guidance potentially provides better diagnostic yield, especially in eccentrically and adjacently orientated lesions due to its capability of lateral biopsy. However, as bleeding is more common with cryobiopsy, this procedure should not be used indiscriminately. More studies in a prospective manner are needed to validate the technique in this group of patients.

Acknowledgement: We would like to express our gratitude and appreciation to the Sarawak Lung Cancer Special Interest Group.

Author contributions: S.S. Kho is the guarantor of the manuscript; S.S. Kho and S.T. Tie had full access to all of the data in the study, and take responsibility for the integrity of the data and the accuracy of the data analysis; S.S. Kho and S.T. Tie conceived and designed the trial and its protocol; S.S. Kho, S.T. Tie, S.K. Chan and M.C. Yong recruitment the patients and collected the data collection; S.S. Kho performed the statistical analyses and interpreted the data; all authors wrote the manuscript and gave final approval.

Conflict of interest: None declared.

\section{References}

1 Callister MEJ, Baldwin DR, Akram AR, et al. British Thoracic Society guidelines for the investigation and management of pulmonary nodules. Thorax 2015; 70: ii1-ii54.

2 National Lung Screening Trial Research Team, Aberle DR, Adams AM, et al. Reduced lung-cancer mortality with low-dose computed tomographic screening. N Engl J Med 2011; 365: 395-409.

3 Chan A, Devanand A, Low SY, et al. Radial endobronchial ultrasound in diagnosing peripheral lung lesions in a high tuberculosis setting. BMC Pulm Med 2015; 15: 90.

4 Wang Memoli JS, Nietert PJ, Silvestri GA. Meta-analysis of guided bronchoscopy for the evaluation of the pulmonary nodule. Chest 2012; 142: 385-393.

5 Ali MS, Trick W, Mba BI, et al. Radial endobronchial ultrasound for the diagnosis of peripheral pulmonary lesions: a systematic review and meta-analysis. Respirology 2017; 22: 443-453.

6 Schuhmann M, Bostanci K, Bugalho A, et al. Endobronchial ultrasound-guided cryobiopsies in peripheral pulmonary lesions: a feasibility study. Eur Respir J 2014; 43: 233-239.

7 Chen AC, Loiselle A, Zhou L, et al. Localization of peripheral pulmonary lesions using a method of computed tomography - anatomic correlation and radial probe endobronchial ultrasound confirmation. Ann Am Thorac Soc 2016; 13: 1586-1592.

8 Kurimoto N, Morita K. Kikanshi-kyō “eda yomi”-jutsu [Reading Technique of Bronchial Branch]. Tokyo: Igako Shoin; 2015.

9 Chen A, Chenna P, Loiselle A, et al. Radial probe endobronchial ultrasound for peripheral pulmonary lesions, a 5-year institutional experience. Ann Am Thorac Soc 2014; 11: 578-582.

10 Hetzel J, Maldonado F, Ravaglia C, et al. Transbronchial cryobiopsies for the diagnosis of diffuse parenchymal lung diseases: expert statement from the cryobiopsy working group on safety and utility and a call for standardization of the procedure. Respiration 2018; 95: 188-200. 
11 Hetzel J, Eberhardt R, Herth FJF, et al. Cryobiopsy increases the diagnostic yield of endobronchial biopsy: a multicentre trial. Eur Respir J 2012; 39: 685-690.

12 Ravaglia C, Bonifazi M, Wells AU, et al. Safety and diagnostic yield of transbronchial lung cryobiopsy in diffuse parenchymal lung diseases: a comparative study versus video-assisted thoracoscopic lung biopsy and a systematic review of the literature. Respiration 2016; 91: 215-227.

13 Gupta A, Youness H, Dhillon SS, et al. The value of using radial endobronchial ultrasound to guide transbronchial lung cryobiopsy. J Thorac Dis 2019; 11: 329-334.

14 Hibare KR, Goyal R, Nemani C, et al. Radial endobronchial ultrasound for the diagnosis of bronchoscopically invisible lesions: first case series from India. Lung India 2017; 34: 43-46.

15 Herath S, Yap E. Novel hybrid cryo-radial method: an emerging alternative to CT-guided biopsy in suspected lung cancer. A prospective case series and description of technique. Respirol Case Rep 2017; 6: e00287.

16 Goyal R, Gogia P, Chachra V. Endobronchial ultrasound-radial probe-assisted cryobiopsy for peripheral lung mass: a new way for better yield? J Bronchology Interv Pulmonol 2016; 23: 67-70.

17 Walter JB, Pryce DM. The site of origin of lung cancer and its relation to histological type. Thorax 1955; 10 $117-126$.

18 Gaeta M, Pandolfo I, Volta S, et al. Bronchus sign on CT in peripheral carcinoma of the lung: value in predicting results of transbronchial biopsy. AJR Am J Roentgenol 1991; 157: 1181-1185.

19 Chao TY, Chien MT, Lie CH, et al. Endobronchial ultrasonography-guided transbrochial needle aspiration increases the diagnostic yield of peripheral pulmonary lesions: a randomized trial. Chest 2009; 136: 229-236.

20 Yarmus LB, Semaan RW, Arias SA, et al. A randomized controlled trial of a novel sheath cryoprobe for bronchoscopic lung biopsy in a porcine model. Chest 2016; 150: 329-336.

21 Berim IG, Saeed AI, Awab A, et al. Radial probe ultrasound-guided cryobiopsy. J Bronchology Interv Pulmonol 2007; 24: 170-173. 